Pacific Journal of Mathematics

A RADON-NIKODÝM THEOREM FOR FINITELY ADDITIVE HUGH BARDEEN MAYNARD 


\title{
A RADON-NIKODYM THEOREM FOR FINITELY ADDITIVE BOUNDED MEASURES
}

\author{
Hugh B. MaYNARD
}

\begin{abstract}
An exact Radon-Nikodym theorem is obtained for finitely additive bounded scalar measures defined on a field, the additional condition being a local condition on the dominant average range. The traditional technique of transferring the problem to the Stone space, which results in approximate Radon-Nikodym derivatives, is circumvented by isolating an Exhaustion principal for finitely additive measures which is then utilized to obtain the necessary decompositions.
\end{abstract}

Examples are given to illustrate the basic difficulties which arise in differentiating with respect to signed finitely additive measures and it is demonstrated that one difficulty arises from a lack of a suitable Hahn decomposition of the differentiating measures. The concept of an exhaustive Hahn decomposition is defined for finitely additive measures and is compared to the related concepts of an approximate Hahn decomposition as well as the standard Hahn decomposition. It is shown that $\mu$ having an exhaustive $\mathrm{Hahn}$ decomposition is equivalent to $|\mu|$ having a Radon-Nikodym derivative with respect to $\mu$ and this result is then applied, in this situation, to obtain a simplified Radon-Nikodym theorem.

The question of characterizing indefinite integrals of finitely additive measures has been under consideration for a number of years. There have been two basic approaches to this problem, both seemingly arising from a desire to characterize the absolutely continuous bounded measures. The first was to enlarge the class of integrable functions to include objects other than point functions and to then obtain an equivalence between absolute continuity and integral representation. Rickart [10] obtained such an equivalence by including the multi-valued contractive set functions, while Tucker and Wayment [12], in the setting of finitely additive operator-valued measures, obtained a similar equivalence between an enlarged class of integrable objects and a generalized definition of absolute continuity. The second approach is that of the Radon-Nikodym Bochner theorem [3, p. 315, Theorem 14] which utilized the Stone space to characterize the absolutely continuous, bounded variation measures as those which can be approximated arbitrarily close in variation by integrals of integrable simple functions. There does not seem to be any characterization of indefinite integrals of point functions with respect to a finitely additive bounded scalar measure prior to 


\section{this paper.}

The method of proof is interesting in that it is shown that if $m$ is representable as an integral with respect to $\mu$, then there exists certain "nice" decompositions of $X$ such that both $\mu$ and $m$ satisfy a restricted form of countable additivity with respect to these decompositions. This is sufficient to allow arguments similar to those used in the Bochner integral case [Maynard, 8, Theorem 2.1]. In fact the lack of various decompositions seems to be the key to the difficulties which arise in the finitely additive situation.

2. An exhaustion principle. The notation and definitions employed in this paper will be the same as those of Dunford and Schwartz [3, Cnapter III] which is an equivalent development, in our setting, to that of Gould [7]. Let $X$ be a set, $\Sigma$ a field of subsets of $X$, and $\mu: \Sigma \rightarrow R$ a finitely additive bounded measure (三 set function). As usual $|\mu|$ will denote the total variation of $\mu$ and is a positive finitely additive measure and $\Sigma^{+}$will denote the subset of $\Sigma$ consisting of sets with positive $\mu$-variation. In addition we will use the notation $\delta(A)$ to denote the diameter of a set $A \subset \boldsymbol{R}$.

Definition 2.1. A set property $P$ is said to be locally exhausting in $(X, \Sigma, \mu)$ if there exists an $\alpha, 0<\alpha \leqq 1$, such that for each $E \in \Sigma^{+}$ there exists $F \subset E, F \in \Sigma^{+}$, such that $|\mu|(F) \geqq \alpha|\mu|(E)$ and $F$ has property $P$.

Definition 2.2. A countable (possibly finite) disjoint collection $\left\{X_{i}\right\}_{i \in I} \subset \Sigma^{+}$is said to be exhausting in $X$ if, given any $\varepsilon>0$, there exists $N>0$ such that

$$
|\mu|\left(X \sim \bigcup_{1=i}^{N} X_{i}\right)<\varepsilon .
$$

LEMMA 2.3 (Exhaustion principle). If $P$ is a locally exhausting set property in $(X, \Sigma, \mu)$, then there exists a countable (possibly finite) set of disjoint subsets, $\left\{X_{i}\right\}_{i \in I} \subset \Sigma^{+}$, such that each $X_{i}$ has property $P$ and $\left\{X_{i}\right\}_{i \in I}$ is exhausting in $X$.

Proof. Since $P$ is locally exhausting, there exists $X_{1} \subset X, X_{1} \in \Sigma^{+}$, such that $X_{1}$ has $P$ and $|\mu|\left(X_{1}\right) \geqq \alpha|\mu|(X)$. Proceed by induction. If $|\mu|\left(X \sim \bigcup_{i=1}^{n} X_{i}\right)=0$, then the process terminates and $\left\{X_{i}\right\}_{i=1}^{n}$ satisfies the conclusions of the lemma. If $|\mu|\left(X \sim \bigcup_{i=1}^{n} X_{i}\right)>0$, choose $X_{n+1} \subset$ $X \sim \bigcup_{i=1}^{n} X_{i}, X_{n+1} \in \Sigma^{+}$, such that $X_{n+1}$ has property $P$ and $|\mu|\left(X_{n+1}\right) \geqq$ $\alpha|\mu|\left(X \sim \bigcup_{i=1}^{n} X_{i}\right)$. If the process never terminates we obtain a disjoint sequence $\left\{X_{i}\right\}_{i=1}^{\infty} \subset \Sigma^{+}$such that each $X_{i}$ has property $P$. 
If $\lim _{n \rightarrow \infty}|\mu|\left(X \sim \bigcup_{i=1}^{n} X_{i}\right) \neq 0$, then there exists a $\beta>0$ such that $|\mu|\left(X \sim \bigcup_{i=1}^{n} X_{i}\right)>\beta$, for $1 \leqq n<\infty$. Thus

$$
|\mu|\left(X_{n}\right) \geqq \alpha|\mu|\left(X \sim \bigcup_{i=1}^{n-1} X_{i}\right)>\alpha \beta>0
$$

for every $n$, and since $\left\{X_{i}\right\}_{i=1}^{\infty}$ is disjoint, this violates the boundedness of $\mu$.

Definition 2.4. A set property $P$ is said to be a null difference property if whenever $E \in \Sigma^{+}$has property $P$ and $F \in \Sigma^{+}$such that $|\mu|(E \Delta F)=0$, then $F$ has property $P$.

Lemma 2.5. $P$ is a locally exhausting null difference property in a complete bounded finitely additive measure space $(X, \Sigma, \mu)$, then there exists a countable (possibly finite) set of disjoint subsets, $\left\{X_{i}\right\}_{i \in I} \subset$ $\Sigma^{+}$, such that $X=\bigcup_{i \in I} X_{i}$, each $X_{i}$ has property $P$, and $\left\{X_{i}\right\}_{i \in I}$ is exhausting in $X$.

Proof. By the Exhaustion principle there exists a set $\left\{X_{i}\right\}_{i \in I}$ satisfying all conclusions except that $X$ need not equal $\cup_{i \in I} X_{i}$. But since $\left\{X_{i}\right\}_{i \in I}$ is exhausting in $X$ we have that $X \sim \cup_{i \in I} X_{i}$ is a $\mu$-null set and hence is measurable by completeness of $(X, \Sigma, \mu)$. Thus since $P$ is a null difference property, $X \sim \bigcup_{i \in I} X_{1}$ may be adjoined to $X_{1}$ without altering any of the desired properties.

3. A Radon-Nikodym theorem. The approach to be used in obtaining a Radon-Nikodym theorem for bounded finitely additive measure is similar to the locally small average range approach for the Bochner integral. The major difficulty in this approach lies in a possible instability of the average range due to locally large values $|\mu|(E) /|\mu(E)|$ of the integrating measure. This is due to the lack of a Hahn decomposition for bounded finitely additive measures. A secondary problem is that while a local property will yield a countable maximal decomposition of the space, the measures need not be countably additive with respect to this decomposition. It is easy to construct examples on the field of finite and cofinite subsets of the integers with locally small average range but without locally exhausting small average range.

We consider first the various types of average ranges which are useful in Radon-Nikodym theorems for the Bochner integral, operator-valued measures, and finitely additive measures. Suppose $m: \Sigma \rightarrow R$ is another finitely additive measure. The standard average range which occurs in the Radon-Nikodym theorem for the Bochner integral [Rieffel [11], Maynard [8]] has the following definition. 
DEFINITION 3.1. For each $E \in \Sigma^{+}$, the average range of $m$ with respect to $\mu$ over $E$ is: $A_{m}(E)=\{m(F) / \mu(F): F \subset E, \mu(F) \neq 0\}$.

However without a Hahn decomposition the local structure of $A_{m}(E)$ may always be poorly behaved when the ratios, $|\mu|(F) /|\mu(F)|$, are large and hence to avoid this problem we consider, with finitely additive measures, the dominant average range.

DEFINITION 3.2. For each $E \in \Sigma^{+}$, the dominant average range of $m$ with respect to $\mu$ over $E$ is

$$
A_{m}^{*}(E)=\left\{m(F) / \mu(F): F \subset E, F \in \Sigma^{+}, \quad \text { and } \quad|\mu(F)|>\frac{1}{2}|\mu|(F)\right\} .
$$

The third average range we will consider is the $\varepsilon$-approximate average range which is useful for operator-valued measures, Maynard [7], but is primarily used here for convienence and to illustrate the connections between the various average ranges.

DEFINITION 3.3. For each $E \in \Sigma^{+}$, the $\varepsilon$-approximate average range of $m$ with respect to $\mu$ over $E$ is

$$
A(E, \varepsilon)=\{x \in \boldsymbol{R}:|m(F)-x \mu(F)| \leqq \varepsilon|\mu|(F), \forall F \subset E, F \in \Sigma\} .
$$

The following two properties are the key properties involved in the Radon-Nikodym theorem for finitely additive measures.

DEFINITION 3.4. $m$ is said to have locally exhausting small dominant average range iff for each $\varepsilon>0$ there exists $\alpha(\varepsilon)>0$ such that for $E \in \Sigma^{+}$there exists $F \subset E, F \in \Sigma^{+}$, with $|\mu|(F)>\alpha(\varepsilon)|\mu|(E)$ and $\delta\left(A_{m}^{*}(F)\right)<\varepsilon$.

DEFINITION 3.5. $m$ is said to have locally exhausting approximate average range iff for each $\varepsilon>0$ there exists $\alpha(\varepsilon)>0$ such that for $E \in \Sigma^{+}$there exists $F \subset E, F \in \Sigma^{+}$, with $|\mu|(F)>\alpha(\varepsilon)|\mu|(E)$ and $A(F, \varepsilon) \neq \varnothing$.

DEFINITION 3.6. If $m, \mu: \Sigma \rightarrow \boldsymbol{R}$ are finitely additive measures, then $m$ is $\mu$-continuous iff for every $\varepsilon>0$ there exists $\delta>0$ such that $|\mu|(E)<\delta$ implies that $|m|(E)<\varepsilon$.

It should be emphasized that the definitions of $\mu$-continuity in [5] and [8], requiring only that $|m(E)|<\varepsilon$, are too restrictive as noted in [4] and should be the above definition.

Lemma 3.7. Let $(X, \Sigma, \mu)$ be a bounded finitely additive measure 
space and $m: \Sigma \rightarrow \boldsymbol{R}$ be a $\mu$-continuous finitely additive measure. Then $m$ has locally exhausting small dominant average range iff $m$ has locally exhausting approximate average range.

Proof. Suppose $m$ has locally exhausting approximate average range, and let $\varepsilon>0$ be given and $\alpha(\varepsilon)$ the guaranteed constant corresponding to $\varepsilon / 4$. Then if $E \in \Sigma^{+}$, there exists $F \subset E, F \in \Sigma^{+}$, $|\mu|(F)>\alpha(\varepsilon)|\mu|(E)$ such that $A(F, \varepsilon / 4) \neq \varnothing$.

Choose $x \in A(F, \varepsilon / 4)$. Then if $F_{1} \subset F, F_{1} \in \Sigma^{+}$, such that $\left|\mu\left(F_{1}\right)\right|>$ $1 / 2|\mu|\left(F_{1}\right)$ we have

$$
\left|\frac{m\left(F_{1}\right)-x}{\mu\left(F_{1}\right)}\right|=\left|m\left(F_{1}\right)-x \mu\left(F_{1}\right)\right| \cdot \frac{1}{\left|\mu\left(F_{1}\right)\right|} \leqq \frac{\varepsilon}{4} \frac{|\mu|\left(F_{1}\right)}{\left|\mu\left(F_{1}\right)\right|}<\frac{\varepsilon}{2} .
$$

Thus $\delta\left(A_{m}^{*}(F)\right)<\varepsilon$ and $m$ has locally exhausting small dominant average range.

Suppose that $m$ has locally exhausting small dominant average range. Let $\varepsilon>0$ be given and $\alpha(\varepsilon)$ the constant corresponding to $\varepsilon$. Then given $E \in \Sigma^{+}$, there exists $F \subset E, F \in \Sigma^{+}$, such that $\delta\left(A_{m}^{*}(F)\right)<$ $\varepsilon$. Choose $F_{1} \subset F$ such that $\left|\mu\left(F_{1}\right)\right|>1 / 2|\mu|\left(F_{1}\right)$. Then it suffices to show that $m\left(F_{1}\right) / \mu\left(F_{1}\right) \in A(F, \varepsilon)$.

Let $C \subset F, C \in \Sigma^{+}$. If $|\mu|(C)=0$ then by $\mu$-continuity, $m(C)=0$ and we have the desired inequality. If $|\mu|(C) \neq 0$, then let $\delta=$ $\min \left(u^{+}(C), \mu^{-}(C)\right)$ where $\mu^{+}(C)=\sup _{D=C} \mu(D)$ and $\mu^{-}(C)=-\inf _{D \subset C} \mu(D)$. If $\delta=0$ the argument is trivial so suppose $\delta>0$. Then by Darst [5] there exist disjoint sets $A, B$ such that $C=A \cup B$ with property that $\mu^{+}(B)<\delta / 4<|\mu|(B) / 4$ and $\mu^{-}(A)<\delta / 4<|\mu|(A) / 4$. Then

$$
|\mu(A)|=\left|\mu^{+}(A)-\mu^{-}(A)\right|>|\mu|(A)-2 \mu^{-}(A)>\frac{|\mu|(A)}{2}
$$

and similarily $|\mu(B)|>|\mu|(B) / 2$. Thus

$$
\begin{aligned}
\left|m(C)-\frac{m\left(F_{1}\right)}{\mu\left(F_{1}\right)} \mu(C)\right|= & \left|m(A \cup B)-\frac{m\left(F_{1}\right)}{\mu\left(F_{1}\right)} \mu(A \cup B)\right| \\
\leqq & \left|m(A)-\frac{m\left(F_{1}\right)}{\mu\left(F_{1}\right)} \mu(A)\right|+\left|m(B)-\frac{m\left(F_{1}\right)}{\mu\left(E_{1}\right)} \mu(B)\right| \\
\leqq & \left|\frac{m(A)}{\mu(A)}-\frac{m\left(F_{1}\right)}{\mu\left(F_{1}\right)}\right||\mu(A)| \\
& +\left|\frac{m(B)}{\mu(B)}-\frac{m\left(F_{1}\right)}{\mu\left(F_{1}\right)}\right||\mu(B)|<\varepsilon|\mu|(A)+\varepsilon|\mu|(B) \\
= & \varepsilon|\mu|(C) .
\end{aligned}
$$

Thus $m\left(F_{1}\right) / \mu\left(F_{1}\right) \in A(F, \varepsilon) \neq \varnothing$ and hence $m$ has locally exhausting approximate average range. As the third example in $\S 4$ demonstrates, 
it is not true that either of these two conditions imply that $m$ has locally exhausting small average range, even if $m$ is an indefinite integral. We are now prepared to prove our main theorem after we point out a restricted form of countable additivity which will enable us to mimic proofs in the countably additive case.

LEMMA 3.8. Let $m$ and $\mu$ be two $R$-valued measures in $(X, \Sigma), \Sigma$ a field, such that $m$ is $\mu$-continuous. Then $\mu$ is uniformly countably additive with respect to a disjoint sequence $\left\{E_{i}\right\}_{i=1}^{\infty} \subset \Sigma$ (i.e., $\forall F \in \Sigma^{+}$, $\mu(F)=\sum_{i=1}^{\infty} \mu\left(F \cap E_{i}\right)$ where convergence is uniform in $\left.F\right)$ iff $\left\{E_{i}\right\}_{i=1}^{\infty}$ is exhausting in $X$. In addition if $\left\{E_{i}\right\}_{i \in I}$ is exhausting in $X$ with respect to $\mu$, then $m$ is also uniformly countably additive with respect to $\left\{E_{i}\right\}_{i=1}^{\infty}$.

The following bound on the $\varepsilon$-approximate average range can easily be calculated.

Lemma 3.9. Let $m$ and $\mu$ be two $R$-valued measures in $(X, \Sigma)$. Then for $\varepsilon>0, \delta(A(E, \varepsilon)) \leqq 2 \varepsilon, E \in \Sigma^{+}$.

THEOREM 3.10 [Radon-Nikodym theorem]. Let $(X, \Sigma, \mu)$ be a bounded finitely additive measure space, $\Sigma$ a field of subsets of $X$ and $\mu$ a signed measure. If $m$ is a finitely additive $\boldsymbol{R}$-valued measure, then there exists a $\mu$-integrable function $f: X \rightarrow \boldsymbol{R}$ such that $m(E)=\int_{E} f d \mu, \forall E \in \Sigma$ iff

(a) $m$ is bounded, $\mu$-continugus and

(b) for all $\delta>0$ there exists $F_{\hat{o}} \subset X, F_{\hat{o}} \in \Sigma$ such that

(i ) $\mu\left(X \sim F_{o}^{\prime}\right)<\delta$

(ii) $A_{m}^{*}\left(F_{\delta}\right)$ is bounded and

(iii) $m$ has locally exhausting small dominated average range in $F_{\hat{o}}$.

Proof. We may assume throughout the proof that $(X, \Sigma, \mu)$ is complete since a function integrable with respect to the completion is integrable with respect to $(X, \Sigma, \mu)$ and has the same integral values.

$\Leftrightarrow$ Suppose $m(E)=\int_{E} f d \mu$. Then (a) is well known [Dunford and Schwartz, 3, III 2.18 and 20]. Let $\delta>0$ be given. Then there exists a simple function $f_{n}$ such that $\mu^{*}\left\{x:\left|f(x)-f_{n}(x)\right|>1\right\}<\delta$. Choose $A \in \Sigma$ such that $A \supset\left\{x:\left|f(x)-f_{n}(x)\right|>1\right\}$ and $\mu(A)<\delta$ and let $F_{\delta}=X \sim A$. Hence $F_{o}$ satisfies (i). Let $N=\sup \left\{\left|f_{n}(x)\right|: x \in F_{\delta}\right\}+1$. Thus $|f(x)| \leqq N$ for all $x \in F_{\hat{o}}$. Now if $E \subset F_{i},|\mu(E)|>1 / 2|\mu|(E)$, then $|m(E)|=\int_{E} f d \mu|\leqq 2 N| \mu|(E) \leqq 4 N| \mu(E) \mid$ and hence $A_{m}^{*}\left(F_{\dot{o}}\right)$ is bounded. 
Let $\varepsilon>0$ be given and let $\alpha(\varepsilon)=\min \{1 / 16, \varepsilon / 8 N\}$ and suppose $E \in \Sigma^{+}, E \subset F_{\delta}$. Since $f$ is totally measurable on $F_{\delta}$, there exists a measurable partition $\left\{X_{i}\right\}_{i=0}^{n}$ of $E$ such that $|\mu|\left(X_{0}\right)<|\mu|(E) / 4$ and $\delta\left(f\left(X_{i}\right)\right)<\varepsilon / 2,1 \leqq i \leqq n$. Now by Lemma 3.7 it suffices to show the equivalence with locally exhausting approximate average range.

Claim 1. $f\left(X_{i}\right) \subset A\left(X_{i}, \varepsilon / 2\right), 1 \leqq i \leqq n$.

Proof. Let $r \in f\left(X_{i}\right)$ and let $F \subset X_{i}, F \in \Sigma^{+}$. Then

$$
|m(F)-r \mu(F)|=\left|\int_{F} f-r d \mu\right| \leqq \frac{\varepsilon}{2}|\mu|(F)
$$

since $|f(x)-r| \leqq \varepsilon / 2$ for all $x \in X_{i}$. Thus $f\left(X_{i}\right) \subset A\left(X_{i}, \varepsilon / 2\right)$.

We now cover the interval $[-N, N]$ with the disjoint intervals $E_{k} \equiv[-N+k \varepsilon / 2,-N+(k+1) \varepsilon / 2), 0 \leqq k \leqq \llbracket 4 N / \varepsilon \rrbracket \equiv Q$ where $\llbracket \cdot \rrbracket$ is the greatest integer function.

For each $k, 0 \leqq k \leqq Q$, we define the following set of indices:

$$
I_{k}=\left\{i: A\left(X_{i}, \varepsilon / 2\right) \cap E_{k} \neq \varnothing\right\} .
$$

Now $A\left(X_{1}, \varepsilon / 2\right)$ must intersect at least one $E_{k}$ since $f\left(X_{i}\right) \subset$ $[-N, N]$ and can intersect no more than two since $\delta\left(A\left(X_{i}, \varepsilon / 2\right)\right) \leqq \varepsilon$.

Claim 2. There exists $k \geqq 0$ such that

$$
|\mu|\left(\bigcup_{i \in \perp_{k}} X_{i}\right)>\alpha(\varepsilon)|\mu|(E) .
$$

Proof. Suppose not. We already know that

$$
|\mu|\left(\bigcup_{i=1}^{n} X_{i}\right) \geqq|\mu|(E)-|\mu|\left(X_{0}\right) \geqq \frac{3|\mu|(E)}{4},
$$

but on the other hand

$$
\begin{aligned}
|\mu|\left(\bigcup_{i=1}^{n} X_{i}\right) & =|\mu|\left(\bigcup_{k=0}^{Q}\left\{\bigcup_{i \in I_{k}} X_{i}\right\}\right) \leqq \sum_{k=0}^{Q}|\mu|\left(\bigcup_{i \in I_{k}} X_{i}\right) \\
& \leqq(Q+1) \alpha(\varepsilon)|\mu|(E) \leqq\left(\frac{4 N}{\varepsilon} \cdot \frac{\varepsilon}{8 N}+\frac{1}{16}\right)|\mu|(E) \\
& \leqq\left[\frac{1}{2}+\frac{1}{16}\right]|\mu|(E)<\frac{3}{4}|\mu|(E) . \quad \Rightarrow=.
\end{aligned}
$$

Thus there exists $I_{k}$ such that $|\mu|\left(\bigcup_{i \in I_{k}} X_{i}\right)>\alpha(\varepsilon)|\mu|(E)$. Let $F=\bigcup_{i \in I_{k}} X_{i}$.

Claim 3. $A(F, \varepsilon) \neq \varnothing$. 
Proof. Let $r=-M+((k+1) / 2) \varepsilon$ and suppose $F^{\prime} \subset F, F^{\prime} \in \Sigma^{+}$. Now for each $X_{i}, i \in I_{k}$, choose $r_{i} \in A\left(X_{i}, \varepsilon / 2\right) \cap E_{k}$.

Then $\left|r-r_{i}\right| \leqq \varepsilon / 2$ since $r, r_{i} \in \bar{E}_{k}$. Now

$$
\begin{aligned}
\mid m\left(F^{\prime}\right) & -r \mu\left(F^{\prime}\right)\left|\leqq \sum_{i \in I_{k}}\right| m\left(F^{\prime} \cap X_{i}\right)-r_{i} \mu\left(F^{\prime \prime} \cap X_{i}\right) \mid \\
& +\sum_{i \in I_{k}}\left|r_{i}-r\right||\mu|\left(F^{\prime} \cap X_{i}\right) \\
\leqq & \sum_{i \in I_{k}} \frac{\varepsilon}{2}|\mu|\left(F^{\prime} \cap X_{i}\right)+\sum_{i \in I_{k}} \frac{\varepsilon}{2}|\mu|\left(F^{\prime} \cap X_{i}\right)=\varepsilon|\mu|\left(F^{\prime}\right) .
\end{aligned}
$$

Thus $r \in A(F, \varepsilon) \neq \varnothing$.

Hence since $|\mu|(F)>\alpha(\varepsilon)|\mu|(E)$ we have finished demonstrating the necessity of our conditions.

$(\Leftarrow)$ Suppose $m$ satisfies (a) and (b) and hence has locally exhausting approximate range.

We will use the following notation. If $z=\left(z_{1}, \cdots, z_{n}\right) \in N^{n}$, then $p(z)=\left(z_{1}, \cdots, z_{n-1}\right), q(z)=z_{n}$, and $(z, i)=\left(z_{1}, \cdots, z_{n}, i\right) \in \boldsymbol{A}^{n+1}$, where the dependence on $n$ is suppressed in an effort for notational simplicity.

Now there exists a disjoint sequence of sets $\left\{F_{N}\right\} \subset \Sigma^{+}$, which of exhausting in $X$, guaranteed by conditions (a) and (b). We will obtain a density for $m$ on each $F_{N}$ and then sum to obtain the entire density. Fix $N$.

Now the set property, $A(F, 1 / 2) \neq \varnothing$, is a locally exhausting null difference property in $F_{N}$ and hence there exists a disjoint countable set $\left\{Y_{z}^{1}\right\}_{z \in A_{1}} \subset \Sigma^{+}, A_{1} \subset N$, with $\left\{Y_{z}^{1}\right\}$ exhausting in $X, F_{N}=$ $\mathrm{U}_{z \in A_{1}} Y_{z}^{1}$, and $A\left(Y_{z}^{1}, 1 / 2\right) \neq \varnothing$.

Since $A\left(F, 1 / 2^{2}\right) \neq \varnothing$ is a locally exhausting null difference property in each $Y_{z}^{1}$ we may decompose each in an exhausting manner, $Y_{z}^{1}=$ $\mathrm{U}_{i \in A_{z}^{2}} Y_{(z, i)}^{2}$, where $A\left(Y_{(z, i)}^{2}, 1 / 2^{2}\right) \neq \varnothing$.

Let $A_{2}=\left\{z \in N^{2}: p(z) \in A_{1}, q(z) \in A_{p(z)}^{2}\right\}$. Thus $F_{N}=\bigcup_{z \in A_{2}} Y_{z}^{2}$ and this decomposition is exhausting.

In general if $\left\{Y_{z}^{n}\right\}_{z \in A_{n}}$ is exhausting in $F_{N}, A_{n} \subset N^{n}, F_{N}=\bigcup_{z \in A_{n}} Y_{z}^{n}$, we may decompose each $Y_{z}^{n}$ in an exhausting manner and obtain the decomposition $\left\{Y_{z}^{n+1}\right\}_{z \in A_{n+1}}$ where

$$
\begin{aligned}
& Y_{z}^{n}=\bigcup_{i \in A_{z}^{n+1}} Y_{(z, i)}^{n+1}, A_{z}^{n+1} \subset N, A\left(Y_{(z, i)}^{n+1}, 1 / 2^{n+1}\right) \neq \varnothing \\
& F_{N}=\bigcup_{z \in A_{n+1}} Y_{z}^{n+1}, A_{n+1}=\left\{z \in N^{n-1}: p(z) \in A_{n}, q(z) \in A_{p(z)}^{n+1}\right\} .
\end{aligned}
$$

We now define a sequence of functions, $f_{n}: F_{N} \rightarrow \boldsymbol{R}$, in the following manner. For each $n$ and each $z \in A_{n}$ choose $x_{z}^{n} \in A\left(Y_{z}^{n}, 1 / 2^{n}\right)$ and let $f_{n}=\sum_{z \in A_{n}} x_{z}^{n} \chi_{Y_{z}^{n}}$.

Claim 1. $f_{n}$ is totally measurable, bounded, and hence integrable 
and $\int_{E} f_{n} d \mu=\sum_{z \in A_{n}} x_{z}^{n} \mu\left(E \cap Y_{z}^{n}\right)$

Proof. Since $\left\{Y_{z}^{n}\right\}_{z \in A_{n}}$ is exhausting in $F_{N}$, the finite sums converge in measure to $f_{n}$ and hence $f_{n}$ is totally measurable. $f_{n}$ is bounded since the dominated average ranges are bounded and hence the 1-approximate average ranges are bounded in $F_{N}$.

Claim 2. $\left\{f_{n}(t)\right\}_{n=1}^{\infty}$ is uniformly Cauchy for $t \in F_{N}$.

Proof. Let $\varepsilon>0$ be given and choose $M$ such that $1 /(2 M)<\varepsilon$.

If $t \in F_{N}$, there exists a sequence $\left\{z_{n}\right\}, z_{n} \in A_{n}$, such that $t \in Y_{z_{n}}^{n}$. Thus if $n, m>M$ with $m>n$ we have that

$$
\begin{aligned}
& f_{n}(t)=x_{z_{n}}^{n} \in A\left(Y_{z_{n}}^{n}, 1 / 2^{n}\right) \subset A\left(Y_{z_{m}}^{m}, 1 / 2^{n}\right) \text { and } \\
& f_{m}(t)=x_{z_{m}}^{m} \in A\left(Y_{z_{m}}^{m}, 1 / 2^{m}\right) \subset A\left(Y_{z_{m}}^{m}, 1 / 2^{n}\right) .
\end{aligned}
$$

But $\delta\left(A\left(Y_{z m}^{m}, 1 / 2^{n}\right)\right) \leqq 1 / 2^{n-1}$ and hence $\left|f_{n}(t)-f_{m}(t)\right| \leqq 1 / 2^{n-1} \leqq 1 / 2^{m}<\varepsilon$ for any $t \in F_{N}$.

We thus can define $g_{N}(t)=\lim _{n \rightarrow \infty} f_{n}(t): F_{N} \rightarrow \boldsymbol{R}$.

Claim 3. $g_{N}$ is totally measurable, bounded and hence integrable.

Proof. $f_{n} \rightarrow g_{N}$ uniformly and hence in measure which implies that $g_{N}$ is totally measurable. $g_{N}$ is bounded since the functions $\left\{f_{n}\right\}$ are uniformly bounded.

Claim 4. $\quad \int_{E} g_{N} d \mu=\lim _{n \rightarrow \infty} \int_{E} f_{n} d \mu, \forall E \in \Sigma, E \subset F_{N}$.

Proof. The functions $\left\{f_{n}\right\}_{n=1}^{\infty}$ are uniformly bounded and converge uniformly, and hence in measure, to $g_{N}$ on $F_{N}$. Thus by the Dominated Convergence theorem we obtain that $g_{N}$ is integrable and $\int_{E} g_{N} d \mu=$ $\lim _{n \rightarrow \infty} \int_{E} f_{n} d \mu, \forall E \in \Sigma$.

Claim 5. $\int_{E} g_{N} d \mu=m(E), \forall E \in \Sigma, E \subset F_{N}$.

Proof. Let $\varepsilon>0$ be given. Then there exists $n$ such that $\left|\int_{E} g_{N} d \mu-\int_{E} f_{n} d \mu\right|<\varepsilon / 2$ and such that $1 / 2^{n}<\varepsilon / 8|\mu|(E)$. Now choose $K>0$ such that

$$
\text { ( i ) }\left|\int_{E} f_{n} d \mu-\sum_{z \leqq\left(K K \mathbb{A}_{n}\right)} x_{z \in}^{n} \mu\left(E \cap Y_{z}^{n}\right)\right|<\frac{\varepsilon}{4}
$$


and

Then

$$
\text { (ii) }|m|\left(E \sim \underset{\left.\substack{z \leqq(K) \ldots K \\ z \in A_{n}}\right)}{\bigcup} Y_{z}^{n}\right)<\frac{\varepsilon}{8} .
$$

$$
\begin{aligned}
& \left|m(E)-\sum_{\substack{z \leqq\left(K \ldots \ldots K \\
z \in A_{n}\right.}} x_{z}^{n} \mu\left(E \cap Y_{z}^{n}\right)\right|
\end{aligned}
$$

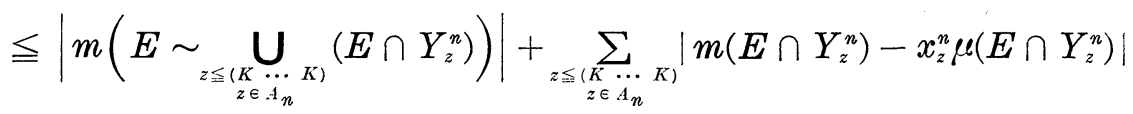

$$
\begin{aligned}
& \leqq \frac{\varepsilon}{8}+\sum_{z \leqq(K, \cdots, K)} \frac{1}{2^{n}}|\mu|\left(E \cap Y_{z}^{n}\right) \quad \text { since } \quad x_{z}^{n} \in A\left(Y_{z}^{n}, 1 / 2^{n}\right) \\
& \leqq \frac{\varepsilon}{8}+\frac{1}{2^{n}}|\mu|(E), \text { since }\left\{Y_{z}^{n}\right\} \quad \text { is exhausting , } \\
& \leqq \frac{\varepsilon}{8}+\frac{\varepsilon}{8}=\frac{\varepsilon}{4} \text {. }
\end{aligned}
$$

Thus

$$
\begin{aligned}
& \left|\int_{E} g_{N} d \mu-m(E)\right| \leqq\left|\int_{E} g_{N} d \mu-\int_{E} f_{n} d \mu\right| \\
& +\left|\int_{E} f_{n} d \mu-\sum_{\substack{z \leqq\left(K \ldots \ldots \\
z \in A_{n}\right.}} x_{z}^{n} \mu\left(E \cap Y_{z}^{n}\right)\right| \\
& +\left|\sum_{z \leqq(K, \cdots, K)} x_{z}^{n} \mu\left(E \cap Y_{z}^{n}\right)-m(E)\right| \\
& \leqq \frac{\varepsilon}{2}+\frac{\varepsilon}{4}+\frac{\varepsilon}{4}=\varepsilon \text {. }
\end{aligned}
$$

Since $\varepsilon>0$ is arbitrary, $\int_{E} g_{N} d \mu=m(E)$.

If we extend each $g_{N}$ to be zero off $F_{N}$ and let $h_{k}=\sum_{N=1}^{k} g_{N}$ and $f=\lim _{k \rightarrow \infty} h_{k}=\sum_{N=1}^{\infty} g_{N}$, it suffices [Dunford and Schwartz, III, 3.6] to show the following three conditions are satisfied.

(i) $h_{k} \rightarrow f$ in measure,

(ii) for each $\varepsilon>0$ there is a $E_{\varepsilon} \in \Sigma$ such that

$$
\int_{X \sim E_{\varepsilon}}\left|h_{k}(s)\right| d|\mu|<\varepsilon, k=1,2, \cdots, \quad \text { and }
$$

(iii) $\lim _{|\mu|\langle E\rangle \rightarrow 0} \int_{E}\left|h_{k}\right| d|\mu|=0$, uniformly in $k$.

The first two conditions follow easily from the exhaustive nature of $\left\{F_{N}\right\}$. If $\varepsilon>0$ is given, choose $\delta>0$ such that $|\mu|(E)<\delta$ implies $|m|(E)<\varepsilon$.

Then for any $k$ and any $E \in \Sigma,|\mu|(E)<\delta$, we have

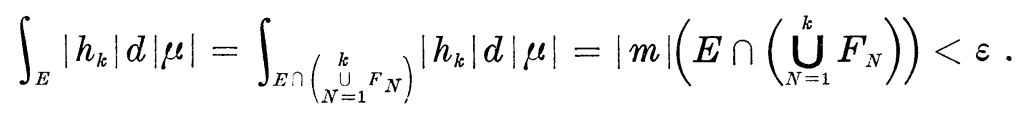


Thus for $E \in \Sigma$,

$$
\int_{E} f d \mu=\sum_{N=1}^{\infty} \int_{E} g_{N} d \mu=\sum_{N=1}^{\infty} m\left(E \cap F_{N}\right)=m(E)
$$

since $\left\{F_{N}\right\}$ is exhausting in $X$.

Corollary 3.11. Let $(X, \Sigma, \mu)$ be a positive bounded finitely additive measure space. If $m$ is a finitely additive $\boldsymbol{R}$-valued measure, then there exists a $\mu$-integrable function $f: X \rightarrow \boldsymbol{R}$ such that $m(E)=\int_{E} f d \mu, \forall E \in \Sigma, i f f$

(a) $m$ is bounded, $\mu$-continuous, and

(b) for all $\delta>0$ there exists $F_{\hat{o}} \subset X, F_{i j} \in \Sigma$ such that

(i) $\mu\left(X \sim F_{i}\right)<\delta$,

(ii) $A_{m}\left(F_{o}\right)$ is bounded and

(iii) $m$ has locally exhausting small average range in $F_{\hat{o}}$.

Proof. If $\mu$ is positive then $\mu=|\mu|$ and hence $A_{m}(E)=A_{m}^{*}(E)$.

4. Examples. The failure of absolute continuity and boundedness to imply the existence of a density arises, it appears, from the lack of appropriate decompositions of the space which are obtainable in the countably additive case on a $\sigma$-algebra.

When the domain is a $\sigma$-algebra, it is impossible to suitably separate the support of countably additive measures and finitely additive measures which yields the failure. If $m$ is Lebesgue measures on $[0,1]$ and $\Sigma$ the Lebesgue measurable subsets of $[0,1]$, we have, for any nonzero $\mu \in\left[L^{\infty}(m)\right]^{*}=b a(\Sigma, m)$ such that $\mu \geqq 0$ and $\mu$ is purely finitely additive, that $m$ is $(m+\mu)$-continuous. However there exists no density $f$ such that $m(E)=\int_{E} f d(m+\mu)=\int_{E} f d m+$ $\int_{E} f d \mu$ since $\int_{E} f d \mu$ must be identically zero, (otherwise it is purely finitely additive) and hence $f=1$ a.e. Thus $\mu \equiv 0$ on $\sum$ which yields the desired contradiction.

If the doman is a field, not a $\sigma$-field, then we can illustrate the failure utilizing countably additive measures since we do not have a Hahn decomposition. Let $X=[0,1), \Sigma$ the field generated by the half open intervals, $[a, b)$. Let $m$ represent Lebesgue measure on $[0,1)$ and choose a Lebesgue measurable set $A \subset[0,1)$ which intersects every interval in a set with positive Lebesgue measure. Define $m(E)=\mu(E \cap A)-\mu\left(E \cap A^{c}\right), E \in \Sigma$. Of course $A \notin \Sigma$. Then $m$ is $\mu$-continuous and $m$ is bounded, in fact $|m|=\mu$. Now $m$ cannot be an indefinite integral with respect to $|m|$ since for $E \in \Sigma^{+}, \delta\left(A_{m}(E)\right)=2$ and hence $m$ does not even have locally small average range. 
A similar example can be used to show that while indefinite integrals need have locally small dominated average range they need not have even locally bounded average range. Let $X, \Sigma, A$, and $m$ be as above and $v(E)=\int_{E} x d m$.

Then if $E \in \Sigma^{+}$, there exists a subset $F \in \Sigma^{+}, F \subset E$, such that $m(F)=0$ and yet $v(F) \neq 0$. Then by $m$-continuity of $v$ there are sets, $\{B\}, B \subset F$ such that the values $\{m(B)\}$ are arbitrarily small and yet $\{v(B)\}$ are uniformly bounded away from zero and hence the average range is never bounded.

The above examples depend upon a lack of suitable decompositions of the underlying space. The effect of appropriate Hahn decompositions is to eliminate many of the difficulties.

DEFINITION 4.1. Let $\mu: \Sigma \rightarrow \boldsymbol{R}$ be a bounded finitely additive measure. Then $\mu$ has a Hahn decomposition iff there exist disjoint sets $A, B \in \Sigma, X=A \cup B$, such that $\mu^{+}(B)=\mu^{-}(A)=0$.

$\mu$ has an approximate Hahn decomposition iff for each $\varepsilon>0$ there exists disjoint sets $A_{\varepsilon}, B_{\varepsilon} \in \Sigma, X=A_{\varepsilon} \cup B_{\varepsilon}$, such that $\mu^{+}\left(B_{\varepsilon}\right)<\varepsilon$ and $\mu^{-}\left(A_{\varepsilon}\right)<\varepsilon$.

$\mu$ has an exhaustive Hahn decomposition iff there exist two increasing sequences $\left\{A_{n}\right\},\left\{B_{n}\right\} \subset \Sigma$ such that $\mu^{+}\left(B_{n}\right)=\mu^{-}\left(A_{n}\right)=0$ and $|\mu|\left(X \sim\left(A_{n} \cup B_{n}\right)\right) \rightarrow 0$ as $n \rightarrow \infty$.

An exhaustive Hahn decomposition is equivalent to the countably additive extension on the Stone space having a Hahn decomposition where each set is, within a null set, a countable union of images from $\Sigma^{+}$. The second example in this section shows that finitely additive bounded measures need not have exhaustive Hahn decompositions. Darst [3, Lemma 2.1] has shown, however, that every finitely additive measure has an approximate Hahn decomposition and, of course, every countably additive measure on a $\sigma$-field has a Hahn decomposition.

The Radon-Nikodym theorem simplifies when the integrating measure has an exhausting Hahn decomposition as the following simple lemmas demonstrate.

LEMмA 4.2. If $\mu$ is a bounded finitely additive measure on $(X, \Sigma), \Sigma$ a field, then there exists a $\mu$-integrable $f$ such that $|\mu|(E)=$ $\int_{E} f d \mu$, iff $\mu$ has an exhaustive Hahn decomposition. If $\Sigma$ is a $\sigma$ field then $|\mu|(E)=\int_{E}$ fd $\mu$ iff $\mu$ has a Hahn decomposition.

LEMma 4.3. If $\mu$ is a bounded finitely additive measure with an exhaustive Hahn decomposition, then any bounded finitely additive 
measure has locally exhaustive small dominated average range with respect to $\mu$ iff it has locally exhaustive small average range.

These lemmas yield the following theorem.

THEOREM 4.4. Let $(X, \Sigma, \mu)$ be a bounded finitely additive measure space with an exhaustive Hahn decomposition. If $m$ is a finitely additive $\boldsymbol{R}$-valued measure, then there exists a $\mu$-integrable function $f: X \rightarrow \boldsymbol{R}$ such that $m(E)=\int_{E} f d \mu, \forall E \in \Sigma$ iff

(a) $m$ is bounded, $\mu$-continuous, and

(b) for all $\delta>0$ there exists $F_{\hat{o}} \subset X, F_{\hat{o}} \in \Sigma$, such that

(i) $\mu\left(X \sim F_{i}\right)<\delta$

(ii) $A_{m}\left(F_{\hat{o}}\right)$ is bounded and

(iii) $m$ has locally exhausting small average range in $F_{\dot{o}}$.

\section{REFERENCES}

1. S. Bochner, Additive set functions on groups, Annals of Math., 40 (1939), 769-799.

2. S. Bochner, and R.S. Phillips, Additive set functions and vector lattices, Annals of Math., 42 (1941), 316-324.

3. N. Dunford and J. T. Schwartz, Linear Operators, Interscience, New York, 1958.

4. R. B. Darst and E. Green, On a Radon-Nikodym theorem for finitely additive set functions, Pacific. J. Math., 27 (1968), 255-259.

5. R. B. Darst, A decomposition of finitely additive set functions, J. Reine and Angenandte Math., 210 (1962), 31-37.

6. C. Fefferman, A Radon-Nikodym theorem for finitely additive set functions, Pacific J. Math., 23 (1967), 35-45.

7. G. G. Gould, Integration over vector-valued measures, Proc. London Math. Soc., 15 (1965), 193-225.

8. H. Maynard, A Radon-Nikodym theorem for operator-valued measures, T.A.M.S., 173 (1972), 449-463.

9. Jan Pachl, An elementary proof of a Radon-Nikodym theorem for finitely additive set functions, P.A.M.S., 32 (1972), 225-228.

10. C. E. Rickart, An abstract Radon-Nikodym theorem, T.A.M.S., 56 (1944), 50-66.

11. M. A. Rieffel, The Radon-Nikodym theorem for the Bochner integral, T.A.M.S., 131 (1968), 466-487.

12. D. H. Tucker and S. G. Wayment, Absolute continuity and the Radon-Nikodym theorem, J. Reine and Angenante Math., 244 (1970), 1-19.

13. K. Yosida and E. Hewitt, Finitely additive measures, T.A.M.S., 72 (1952), 46-66.

Received February 20, 1979.

The University of Texas at San antonio

SAN ANTONio TX 78285 



\title{
PACIFIC JOURNAL OF MATHEMATICS
}

\section{EDITORS}

DONALD BABBITT (Managing Editor)

University of California

Los Angeles, CA 90024

\section{HUGO RossI}

University of Utah

Salt Lake City, UT 84112

C. C. MOORE and ANDREW OGG

University of California

Berkeley, CA 94720

\section{J. DUGUNDJI}

Department of Mathematics University of Southern California Los Angeles, CA 90007

R. FINN and J. Milgram

Stanford University Stanford, CA 94305

\section{ASSOCIATE EDITORS}
E. F. BECKENBACH
B. H. NeUmanN
F. WOLF
K. YosHIDA

\section{SUPPORTING INSTITUTIONS}

\author{
UNIVERSITY OF BRITISH COLUMBIA \\ CALIFORNIA INSTITUTE OF TECHNOLOGY \\ UNIVERSITY OF CALIFORNIA \\ MONTANA STATE UNIVERSITY \\ UNIVERSITY OF NEVADA, RENO \\ NEW MEXICO STATE UNIVERSITY \\ OREGON STATE UNIVERSITY \\ UNIVERSITY OF OREGON
}

\author{
UNIVERSITY OF SOUTHERN CALIFORNIA \\ STANFORD UNIVERSITY \\ UNIVERSITY OF HAWAII \\ UNIVERSITY OF TOKYO \\ UNIVERSITY OF UTAH \\ WASHINGTON STATE UNIVERSITY \\ UNIVERSITY OF WASHINGTON
}

The Supporting Institutions listed above contribute to the cost of publication of this Journal, but they are not owners or publishers and have no responsibility for its content or policies.

Mathematical papers intended for publication in the Pacific Journal of Mathematics should be in typed form or offset-reproduced, (not dittoed), double spaced with large margins. Please do not use built up fractions in the text of the manuscript. However, you may use them in the displayed equations. Underline Greek letters in red, German in green, and script in blue. The first paragraph or two must be capable of being used separately as a synopsis of the entire paper. Please propose a heading for the odd numbered pages of less than 35 characters. Manuscripts, in triplicate, may be sent to any one of the editors. Please classify according to the scheme of Math. Reviews, Index to Vol. 39. Supply name and address of author to whom proofs should be sent. All other communications should be addressed to the managing editor, or Elaine Barth, University of California, Los Angeles, California, 90024.

50 reprints to each author are provided free for each article, only if page charges have been substantially paid. Additional copies may be obtained at cost in multiples of 50 .

The Pacific Journal of Mathematics is issued monthly as of January 1966. Regular subscription rate: $\$ 84.00$ a year (6 Vols., 12 issues). Special rate: $\$ 42.00$ a year to individual members of supporting institutions.

Subscriptions, orders for numbers issued in the last three calendar years, and changes of address should be sent to Pacific Journal of Mathematics, P.O. Box 969, Carmel Valley, CA 93924, U.S.A. Older back numbers obtainable from Kraus Periodicals Co., Route 100, Millwood, NY 10546.

PUBLISHED BY PACIFIC JOURNAL OF MATHEMATICS, A NON-PROFIT CORPORATION

Printed at Kokusai Bunken Insatsusha (International Academic Printing Co., Ltd.). 8-8, 3-chome, Takadanobaba, Shinjuku-ku, Tokyo 160, Japan.

Copyright (C) 1979 by Pacific Journal of Mathematics Manufactured and first issued in Japan 


\section{Pacific Journal of Mathematics}

\section{Vol. 83, No. $2 \quad$ April, 1979}

Patrick Robert Ahern, On a theorem of Hayman concerning the derivative of a

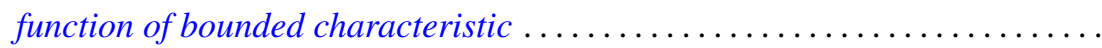

Walter Allegretto, Finiteness of lower spectra of a class of higher order elliptic

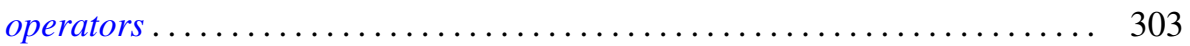

Leonard Asimow, Superharmonic interpolation in subspaces of $C_{c}(X) \ldots \ldots 11$

Steven F. Bellenot, An anti-open mapping theorem for Fréchet spaces . . . . . . . 325

B. J. Day, Locale geometry. . . . . . . . . . . . . . . . . . . . . . . . . . 333

John Erik Fornaess and Steven Krantz, Continuously varying peaking

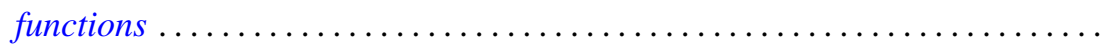

Joseph Leonide Gerver, Long walks in the plane with few collinear points ......

Joseph Leonide Gerver and Lawrence Thom Ramsey, On certain sequences of

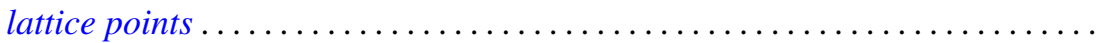

John R. Graef, Yuichi Kitamura, Takaŝi Kusano, Hiroshi Onose and Paul Winton

Spikes, On the nonoscillation of perturbed functional-differential

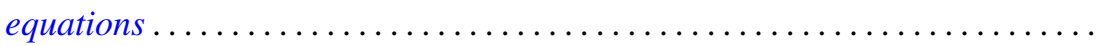

James A. Huckaba and James M. Keller, Annihilation of ideals in commutative

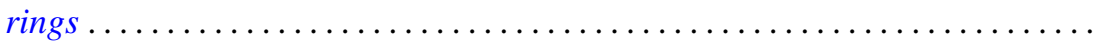

Anzelm Iwanik, Norm attaining operators on Lebesgue spaces . . . . . . . . . . .

Surjit Singh Khurana, Pointwise compactness and measurability . . . .......... 387

Charles Philip Lanski, Commutation with skew elements in rings with

involution.

Hugh Bardeen Maynard, A Radon-Nikodým theorem for finitely additive bounded

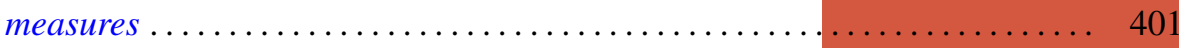

Kevin Mor McCrimmon, Peirce ideals in Jordan triple systems ..

Sam Bernard Nadler, Jr., Joseph E. Quinn and N. Stavrakas, Hyperspaces of compact convex sets.

Ken Nakamula, An explicit formula for the fundamental units of a real pure

sextic number field and its Galois closure ............

Vassili Nestoridis, Inner functions invariant connected components . .

Vladimir I. Oliker, On compact submanifolds with nondegenerate parallel

normal vector fields.

Lex Gerard Oversteegen, Fans and embeddings in the plane.

Shlomo Reisner, On Banach spaces having the property G.L

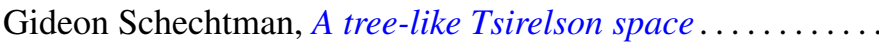

Helga Schirmer, Fix-finite homotopies . . . . . . . . . . . .

Jeffrey D. Vaaler, A geometric inequality with applications to linear forms . .

William Jennings Wickless, $T$ as an $\mathscr{G}$ submodule of $G$.....

Kenneth S. Williams, The class number of $Q(\sqrt{-p})$ modulo 4 , for $p \equiv 3$ (

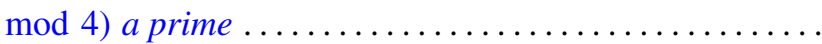

